predominantly grey matter, and larger lesion volume and poorer organisation of the white matter. It is hypothesised that subclinical cardiac malfunction may be responsible for the brain changes, and that reductions in blood volume may cause cerebral microvascular damage or interfere with the function of the blood-brain barrier, resulting in brain damage.

Zonneveld, H. I., Arfan Ikram, M., Hofman, A., et al (2016)

$\mathrm{N}$-terminal pro-B-type natriuretic peptide and subclinical brain damage in the general population. Radiology. https://doi. org/10.1148/radiol.2016160548.

\section{Eat hot red chili pepper and live longer}

Tf you are one of the people who love spicy hot food, you can rejoice! A large populationbased cohort study from the National Health and Nutritional Examination Survey (NHANES) III, a representative sample of US non-institutionalised adults, in which participants were surveyed from 1988 to 1994, examined the relationship between consumption of hot red chili peppers and mortality. Those who consumed them had a $13 \%$ lower risk of death than those who did not. Despite some limitations in this study, the finding is in keeping with some previous studies showing the beneficial effects of spices and in this case capsaicin, contained in hot red chili peppers.

One mechanism for these beneficial effects may be an increase in lipid catabolism in different tissues protecting against hypercholesterolaemia and obesity, hence reducing the risk of type 2 diabetes and atherosclerotic cardiovascular disease. There may also be antimicrobial effects, such as against $H$. pylori, and beneficial changes in the gut microbiota, as well as anti-inflammatory, antioxidant and other possible benefits.

A word of warning though: by all means eat red hot chili to your heart's content but don't throw away your statins! Not yet!

Chopin, M., Lichtenberg, B. \& Gualillo, O. (2017) The association of hot red chili pepper consumption and mortality: a large population-based cohort study. PLoS One, 12, e0169876. https:// doi.org/10.1371/journal.pone.0169876.

\section{Drug-using mothers - and fathers}

re drug-using mothers wholly responsible for A the damage to their offspring's development? Is it time for fathers to take some responsibility too? Research on the development of children's brains, and their health in general, has focused so far on the mother's health and substance misuse. But what about the father? A recent study by a team of researchers from the Perelman School of Medicine, University of Pennsylvania, examined the possibility of fathers' contribution to the developmental problems of their offspring, in rats. They examined drug-naïve rat offspring of cocaine-using fathers and found profound effects on the physiology and behaviour of the males (but not the females). They showed impairment in memory formation associated with reductions in NMDA receptor-mediated hippocampal synaptic plasticity. Concentrations of $\mathrm{d}$-serine, an endogenous NMDA receptor co-agonist, essential for memory, were decreased. Administration of d-serine in the hippocampus reversed both the memory formation deficits and synaptic plasticity. The authors concluded that paternal cocaine exposure causes epigenetic remodelling in the hippocampus of their male progeny.

Wimmer, M. E., Briand, L. A., Fant, B., et al (2017) Paternal cocaine taking elicits epigenetic remodeling and memory deficits in male progeny. Molecular Psychiatry. Published online 21 February. https:// doi.org/10.1038/mp.2017.8

\section{'I would never want to have a mental health diagnosis on my record'}

Dandora has previously reported on the low Prates of seeking treatment for mental health problems among doctors. Medical boards in most US states require disclosure of mental health problems on licensing applications. Does this increase stigma and discourage physicians from seeking help for mental health problems? Is this unique to US doctors?

A survey was carried out via a closed Facebook group of over 2000 female physician-parents. Almost half of these believed themselves to meet diagnostic criteria for mental illness but had not sought help. Only $6 \%$ of physicians with a diagnosis and treatment of mental disorder had disclosed this. The main reasons reported for failing to seek help were that they could manage without help, had no time to do so, fear of reporting to a licensing board and embarrassment and shame.

How can we persuade patients to be comfortable with their mental health problems when we are not? Should there be a review of the regulatory systems for doctors?

Gold, K. J., Andrew, L. B., Goldman, E. B., et al (2016) 'I would never want to have a mental health diagnosis on my record': a survey of female physicians on mental health diagnosis, treatment, and reporting. General Hospital Psychiatry, 43, 51-57. http://dx.doi. org/10.1016/j.genhosppsych.2016.09.004

\section{Depressive disorders the single largest contributor to non-fatal health loss}

Despite all efforts to improve the recognition and treatment of depression, new estimates from the World Health Organization (WHO), released on 23 February this year, show an $18 \%$ increase between 2005 and 2015 in the number of people living with depression. This reflects the overall increase in the global population, as well as a proportionate rise in the age groups in which depression is more prevalent. Globally, depressive disorders are now ranked as the single largest contributor to non-fatal health loss, at $7.5 \%$ of all 'years lost due to disability' (YLD). More than $80 \%$ of the burden of depression is among people in low- and middle-income countries. Rates vary across WHO regions, from 640 YLD per 100000 population in the Western Pacific Region to over 850 in low- and middle-income countries of the European Region. Prevalence rates also vary by age, peaking in older adulthood (above $7.5 \%$ among females aged 55-74 years, and above $5.5 \%$ among males). Depression also occurs in children and adolescents below the age of 15 years, but at a lower level than among older age groups.

World Health Organization global health estimates (http://www. who.int/healthinfo/global_burden_disease). 